\title{
A mass media programme to prevent smoking among adolescents: costs and cost effectiveness
}

Roger H Secker-Walker, John K Worden, Robert R Holland, Brian S Flynn, Allan S Detsky

\begin{abstract}
Objective-To examine costs and costeffectiveness ratios of a four-year mass media programme previously shown to prevent the onset of smoking among adolescents.

Design-A matched control design.

Setting-Two cities in Montana, one in New York and one in Vermont, USA.

Subjects-Students in grades 10-12 (ages 15-18).
\end{abstract}

Intervention-A four-year mass media campaign to prevent the onset of smoking. Main outcome measures-Cost per student potentially exposed to the mass media campaign; cost per student smoker potentially averted; and cost per life-year gained. Cost estimates were also made for a similar campaign that would be broadcast nationally in the United States. Results-In 1996 dollars, the cost of developing and broadcasting the mass media campaign was $\$ 759436$, and the cost per student potentially exposed to the campaign ( $n=18600)$ was $\$ 41$. The cost per student smoker averted $(n=1023)$ was $\$ 754$ (95\% confidence interval $(\mathrm{CI})=\$ 531-$ $\$ 1296)$. The cost per life-year gained discounted at $3 \%$ over the life expectancy for young adult smokers was $\$ 696(95 \%$ $C I=\$ 445-\$ 1269)$. The estimated cost of developing and broadcasting a similar four-year mass media campaign in all 209 American media markets would be approximately $\$ 84.5$ million, at a cost of $\$ 8$ per student potentially exposed to a national campaign, $\$ 162$ per student smoker averted, and $\$ 138(95 \% \mathrm{CI}=$ \$88-\$252) per life-year gained.

Conclusion-Estimates of the costeffectiveness ratios of this mass media campaign in preventing the onset of smoking showed it to be economically attractive and to compare favourably with other preventive and therapeutic strategies.

(Tobacco Control 1997;6:207-212)

Keywords: smoking prevention; mass media; cost effectiveness; adolescents

\section{Introduction}

Recent estimates put the direct and indirect costs of cigarette smoking at about $\$ 68$ billion per year. ${ }^{1}$ In their detailed international review of smoking prevention programmes, Reid et al discuss the costs of implementing a variety of smoking prevention programmes, but do not address the cost effectiveness of these programmes. ${ }^{2}$ Smoking cessation programmes have been shown to be economically attractive, ${ }^{3-5}$ but we are not aware of published estimates for cost-effectiveness ratios of smoking prevention programmes.

Health-related cost-benefit analyses com- i pare the monetary cost of developing and implementing a given programme with the monetary value of the short-term and long-term benefits of the programme. If none, or very few, of the principal benefits expected from a programme can be given a monetary value, cost-effectiveness analysis is more appropriate. ${ }^{6}$ In these situations, the denominator of the cost-effectiveness ratio, the net health effectiveness, can be measured in several ways. For example, when comparing different smoking cessation interventions, the number of successful quitters could be used. ${ }^{7}$ A more general approach, estimating years of life gained or quality-adjusted years of life gained, allows comparisons across a wide range of preventive and therapeutic services. ${ }^{8}$

Because of the substantial difference in life expectancy between non-smokers and smokers, with estimates ranging from 0.2 to 8.6 years, ${ }^{9-17}$ the cost effectiveness of smoking prevention programmes in terms of life-years saved might compare favourably with other preventive and therapeutic measures. ${ }^{918-20}$ This paper provides estimates of the costs and costeffectiveness ratios of a successful four-year mass media intervention to prevent the onset of cigarette smoking ${ }^{21-23}$ from the perspective of organisations that might implement such programmes in the future.

\section{Methods}

Our estimates of the costs and costeffectiveness ratios of a smoking prevention programme are based on a project that took place between 1984 and 1991 in four communities, two in Montana and two in the northeastern United States. The project was designed to test the effect of an intensive mass media programme (television and radio) added to a school smoking prevention curriculum. Two communities, one in Montana and one in the northeastern United States, received the mass media and school programmes, whereas the other two communities received the school programme alone for four years, beginning in grades 5-7 (ages 10-13) and continuing through grades 8-10 (ages 13-16). Both the mass media programme and smoking prevention curriculum had the same educational objectives. Details of the \\ Medicine, Mount Sinai \\ Canada \\ A S Detsky

Office of Health
Promotion Research
and the Vermont
Cancer Center,
Burlington, Vermont,
USA
R H Secker-Walker
J K Worden
B S Flynn
Department of Family
Practice, University of
Vermont
B S Flynn
North Country
Hospital, Newport,
Vermont
R R Holland
Department of
Medicine, Mount Sinai
Hospital, Toronto,
Canada
A S Detsky
Correspondence to:
R H Secker-Walker, 1 South
Prospect Street, Burlington,
Vermont 05401, USA; email:
rseckerw@gnu.uvm.edu


Table 1 Prevalence of weekly smoking for all students and by gender in 1991

\begin{tabular}{lccc}
\hline & \multicolumn{3}{c}{ Smoked in past week } \\
\cline { 2 - 4 } Samples & $n$ & $\%$ & $95 \%$ CI \\
\hline All students & & & \\
$\quad$ Mass media and school programme & 2335 & 20.4 & $18.7-22.0$ \\
School programme only & 2851 & 25.9 & $24.3-27.6$ \\
$\quad$ Difference & & 5.5 & $3.2-7.8$ \\
Girls* & 1151 & 21.0 & $18.7-23.3$ \\
Mass media and school programme & 1327 & 28.3 & $25.9-30.6$ \\
School programme only & & 7.3 & $3.9-10.7$ \\
Difference & & & $17.5-22.1$ \\
Boys* & 1166 & 19.8 & $21.7-25.9$ \\
Mass media and school programme & 1521 & 23.8 & $0.9-7.1$ \\
School programme only & & 4.0 & \\
Difference & & & \\
\hline
\end{tabular}

*The sample sizes for girls and boys do not sum to the total sample size because of missing observations for gender.

$\mathrm{CI}=$ confidence interval. the school programme-only communities for all students and for girls and boys separately is shown in table 1 . The differences for all students and for girls and boys separately were all significant, $\mathrm{P}<0.05$. The confidence intervals shown are based on simple random sampling. The significance of these differences, and the sizes of the confidence intervals, were not changed when community, rather than individual, was considered the primary sampling unit because of an estimated negative variance component associated with community.

Because the same smoking prevention curriculum was implemented in the schools in all four communities, the differences in smoking prevalence represent the incremental effect of adding the mass media programme to the school smoking prevention curriculum and serve as our measure of the efficacy of the mass media programme.

the intervention, and efficacy of the mass media campaign after four years of broadcasting and two years later, have been described previously. ${ }^{21-23}$

The mass media component consisted of 36 television spots and 17 radio spots specifically designed to appeal to students as they passed through adolescence. ${ }^{22}$ The spots aired initially were those prepared for students in grades 5-7. Over the next three years, new spots, designed to appeal to these students as they matured through grades 8-10, were added and some of the earlier ones were dropped. The smoking prevention curriculum, four lessons per year per grade for grades 5-9 (ages 10-15), and three lessons for grade 10 (ages 15-16), ${ }^{25}$ was designed to be typical of smoking prevention curricula that would be available in the United States in the 1990 s, and was taught by regular classroom teachers in all four communities. These teachers were trained in the curriculum's use during day-long workshops each year, starting with grades 5-7 in 1985, and adding a grade each year through 1988 .

Students were surveyed in school by the University of Vermont's Biometry Facility survey team at baseline in May 1985, and then each year for the four years of the interventions (1986-89) and again two years after completion of the interventions (1991), as described previously. ${ }^{21}{ }^{23}$ The efficacy of the mass media campaign was determined at two points in time: in 1989, immediately after four years of the mass media campaign, when the target group was in grades $8-10,{ }^{21}$ and again in 1991 , when the target group was in grades 10-12 (ages 15-18). ${ }^{23}$

To estimate cost-effectiveness ratios, we used the difference in smoking prevalence in the seventh year of the project between all students in grades $10-12$ in the two mass media/school programme communities and all students in these grades in the two school programme communities. The students surveyed in these grades included those who had been at these schools since the inception of the project and thus potentially exposed to the entire campaign, and others who had transferred to them since then, who would have had less potential exposure.

Smoking prevalence in 1991 in the mass media/school programme communities and in

\section{COST ESTIMATES} costs for the mass media programme were derived from our records. ${ }^{21}{ }^{22}$ These costs include: salary and fringe benefits for research staff, consultant fees, travel costs, and costs of data entry and analysis for the diagnostic and formative research; and the preliminary and final production costs of the television and radio spots completed by six media producers. We have inflated these costs by applying the average annual increase in the All Items Consumer Price Index (CPI) between 1980 and $1991,4.43 \%$ per year, to obtain cost estimates for $1996 .^{27}$ For air-time costs, we estimated the 1996 costs of mounting an identical four-year media campaign directly from 1996 rates quoted by the television and radio stations in the two mass media/school programme communities.

Exposure to the mass media programme. Because far more students were potentially exposed to the mass media programme than were surveyed, we estimated the number of those potentially exposed in the two mass media/school programme communities. This estimate, 18600 students for the three-year cohort, was derived from area-of-dominantinfluence tables, ${ }^{28}$ which rank the media markets in the United States by number of households reached by each market, and from 1990 United States census data. ${ }^{29}$

\section{COST-EFFECTIVENESS RATIO}

We estimated the cost-effectiveness ratio for the mass media programme from the perspective of an organisation mounting a similar campaign in 1996. We used a cohort Markov model with annual cycles to determine life expectancy differences between populations with balanced gender ratios but differing prevalence of cigarette smoking. ${ }^{30}$

Mortality rates were determined from life tables for female and male never-smokers, former smokers, and "light" and "heavy" smokers in five-year age categories from 25-29 years to $>75$ years, obtained from a published analysis of 18733 deaths. ${ }^{16}$ We combined the categories of never-smokers and former smok-
The original development and production 
ers to characterise non-smokers, and "light" and "heavy" smokers to characterise smokers. The ratio of "light" and "heavy" smokers we used was representative of that found in the general population.

Our model begins at age 20, and for the first five years allows no mortality difference between populations, but applies discounting to the life-years gained. At age 25 a difference between populations in mortality rate due to cigarette smoking begins and is varied appropriately for age until less than 0.0001 years of additional life expectancy are accrued annually. Beginning at age 75 we assumed a constant annual mortality rate to provide the requisite remaining life expectancy. The life expectancy at age 20 for smokers was found to be an additional 58.97 years for women and 50.17 years for men; whereas that for non-smokers was an additional 64.03 years for women and 56.50 for men. The female smoker loses 5.06 years of life expectancy and the male smoker 6.33 years.

The prevalence of smoking and the efficacy of the mass media programme are shown in table 1 . To assess the effect of variation in the efficacy of the mass media campaign within its $95 \%$ confidence intervals, we performed a Monte Carlo simulation with 1000 runs assuming a normal distribution of this variable and the standard deviation derived from the confidence intervals presented in table $1 .^{31}$

\section{SENSITIVITY ANALYSES}

We examined the effect of different discount rates; different mass media effect sizes, based on the $95 \%$ confidence intervals of the observed differences; different per student costs; halving the years of life lost because of smoking; and also variations in the prevalence of smoking, ranging from 0.5 to 1.5 times the observed prevalence.

\section{NATIONAL CAMPAIGN}

To estimate the number of children, ages 10-12 years, in all 209 media markets in the United States, we used area-of-dominantinfluence tables to identify six large, six medium, and 12 small media markets. ${ }^{28}$ Area-of-dominant-influence tables rank the media markets in the United States by number of households, rounded to the nearest hundred, reached by each market. Maps of each area of dominant influence show the counties within each area and the number of households in each county. We used the tables to randomly select six markets from the first 40 largest markets, six from the next 80 mediumsize markets, and 12 from the remaining 89 small markets. We then used 1990 census data to estimate the average proportion of children, ages 10-12 years, living in the households in the counties for the large, medium, and small markets. We then applied these proportions to the total number of households in the 40 largest markets, the 80 medium-size markets, and the 89 small markets to obtain estimates of the total number of children, ages 10-12, in these markets.
To estimate the costs of broadcasting a -1 national, four-year mass media campaign simi- 음 lar to the one we implemented in 1986-89, we ? obtained quotes of 1996 rates from advertising

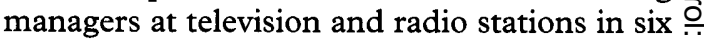
markets: two of the randomly selected large markets, ranked number 4 with $2661800 \stackrel{\text { ? }}{?}$ households and number 27 with 886600 households; two medium-size markets, ranked $\frac{\bar{\sigma}}{\overline{0}}$ number 64 with 407600 households, and $\vec{\nabla}$ number 93 with 271300 households; and two small markets, ranked number 135 with के 158400 households and number 182 with $\vec{\circ}$ 65800 households. ${ }^{28}$ We used these rates and the total number of minutes in prime time, $\vec{\omega}$ early evening "fringe" time, late afternoons, $\stackrel{?}{?}$ and Saturday mornings purchased in the origi- os nal campaign to estimate the costs of $\omega$ broadcasting an identical mass media $\bigcirc$ campaign in each of these markets in 1996. We 을 assumed that other markets of similar size would have similar costs. We used the estimate $\mathcal{P}$ for the market ranked number 4 for the first 20 ? markets, number 27 for markets 21-40, number 64 for markets 41-80, number 93 for markets 81-120, number 135 for markets $121-160$, and number 182 for markets $\ddot{\theta}$ 161-209. We summed these to obtain the 1996 . cost for a sample broadcast year. We multiplied this estimate by four to obtain the total cost of broadcasting the four-year mass media campaign.

\section{Results}

The message development and production costs of the 36 television spots totalled $\$ 373284$, for an average of $\$ 10369$ per spot. Total costs for the 17 radio spots were $\$ 34000$, for an average of $\$ 2000$ per spot. Thus, the overall development and production costs for the mass media campaign were $\$ 407284$. Of this total, $\$ 225124$ was for the diagnostic and formative research that went into message development and broadcast planning, and $\$ 182160$ was for preliminary and final production of the television and radio spots. Inflated by the average annual increase in the CPI, development and production costs would be $\$ 576102$ in 1996 .

Air-time was purchased on local television and radio stations to ensure that the spots or would be broadcast at times that the target $N$ audience was likely to be watching television or N listening to the radio, as indicated by the results of our annual school surveys. The total broadcast cost for the two communities receiving the mass media intervention over four years, at $\stackrel{\mathcal{D}}{?}$ 1996 rates, would have been $\$ 183334$. 궁 Combining the costs of development, production, and broadcasting gave a total fouryear programme implementation cost of $\stackrel{\mathbb{Q}}{\unrhd}$ $\$ 759436$ in 1996 dollars.

COSTS PER SMOKER AVERTED

As table 1 shows, the prevalence of weekly smoking among 2335 students in grades 10-12 who were surveyed in the two media/school programme communities was $20.4 \%$ in 1991 . In the school-only communities, the prevalence of weekly smoking was $25.9 \%$, for a 
Table 2 Cost-effectiveness ratios of the mass media intervention in 1996 dollars

\begin{tabular}{llllll}
\hline & \multicolumn{2}{c}{ Broadcast only } & & \multicolumn{2}{c}{ Development and broadcast } \\
\cline { 2 - 3 } $\begin{array}{l}\text { Discount } \\
\text { rate } \\
(\%)\end{array}$ & $\begin{array}{l}\text { Cost per life-year } \\
\text { gained } \\
(\$)\end{array}$ & $\begin{array}{l}95 \% C I \\
(\$)\end{array}$ & & $\begin{array}{l}\text { Cost per life-year } \\
\text { gained } \\
(\$)\end{array}$ & $\begin{array}{l}95 \% \text { CI } \\
(\$)\end{array}$ \\
\hline 0 & 32 & $21-54$ & & 133 & $88-229$ \\
3 & 167 & $107-301$ & & 696 & $445-1269$ \\
5 & 417 & $266-747$ & & 1732 & $1118-3333$ \\
\hline
\end{tabular}

Broadcast-only cost in 1996 dollars: $\$ 183334$, or $\$ 9.86$ per student

Development and broadcast cost in 1996 dollars: $\$ 759436$, or $\$ 40.83$ per student

$\mathrm{CI}=$ confidence interval.

difference of 5.5 percentage points $(95 \% \mathrm{CI}=$ 3.2 to 7.8 ). This difference represents an estimated $128(95 \% \mathrm{CI}=75$ to 182$)$ fewer students beginning to smoke among those surveyed in the two media/school programme communities. Thus, from the perspective of the research project, the cost per student fully or partially exposed to the media campaign was $\$ 759436 / 2335=\$ 325$, and the cost per student smoker averted was $\$ 759436 / 128=$ $\$ 5933$ (95\% CI $=\$ 4173$ to $\$ 10126)$.

From the perspective of an organisation mounting a mass media campaign, it is important to estimate the cost for all the students in the targeted grades in the two communities exposed to the media/school programme, not just those surveyed for programme evaluation purposes. The cost per student in grades 5-7 who were potentially exposed to the media programme in these communities would be $\$ 759436 / 18600=\$ 41$ for the four-year campaign. Assuming that the media campaign was associated with the same proportional reduction in the onset of smoking among these students ( 5.5 percentage points), there would be $18600 \times 0.055=1023(95 \%$ CI 595 to 1451) fewer smokers, for a cost per student smoker averted of $\$ 759436 / 1023=\$ 742$ ( $95 \%$ CI $\$ 523$ to $\$ 1276$ ).

For an already developed media campaign, the costs would be those required to purchase air-time only, $\$ 183334$. Thus, the cost per student in grades 5-7 who were potentially exposed to the media programme would be $\$ 183334 / 18600=\$ 10$, and the cost per student smoker averted would be $\$ 183334 /$ $1023=\$ 179$ (95\% CI $=\$ 126$ to $\$ 308)$.

Table 3 Cost-effectiveness ratios of the mass media intervention for a range of smoking prevalence

\begin{tabular}{|c|c|c|c|c|c|c|}
\hline \multirow[b]{2}{*}{$\begin{array}{l}\text { Adjustment } \\
\text { in smoking } \\
\text { prevalence }\end{array}$} & \multicolumn{2}{|c|}{ Smoking prevalence } & \multicolumn{2}{|c|}{ Broadcast only } & \multicolumn{2}{|c|}{$\begin{array}{c}\text { Development and } \\
\text { broadcast }\end{array}$} \\
\hline & $\begin{array}{l}\text { School } \\
\text { programme } \\
(\%)\end{array}$ & $\begin{array}{l}\text { Medial } \\
\text { school } \\
\text { programme } \\
(\%)\end{array}$ & $\begin{array}{l}\text { Cost/ } \\
\text { life-year } \\
\text { gained } \\
(\$)\end{array}$ & $\begin{array}{l}95 \% C I \\
(\$)\end{array}$ & $\begin{array}{l}\text { Cost/ } \\
\text { life-year } \\
\text { gained } \\
(\$)\end{array}$ & $\begin{array}{l}95 \% C I \\
(\$)\end{array}$ \\
\hline 0.50 & 13.0 & 10.2 & 335 & $214-637$ & 1415 & $912-2654$ \\
\hline 0.75 & 19.4 & 15.3 & 224 & $147-407$ & 913 & $598-1613$ \\
\hline 0.90 & 23.3 & 18.4 & 187 & $119-339$ & 787 & $500-1488$ \\
\hline 1.00 & 25.9 & 20.4 & 167 & $107-301$ & 696 & $445-1269$ \\
\hline 1.10 & 28.5 & 22.4 & 155 & $100-271$ & 628 & $417-1124$ \\
\hline 1.25 & 32.4 & 25.5 & 134 & $87-239$ & 557 & $367-1057$ \\
\hline 1.50 & 38.9 & 30.6 & 111 & $72-208$ & 464 & $302-853$ \\
\hline
\end{tabular}

Adjustment in smoking prevalence is the multiplier used to adjust the observed prevalence, shown in bold.

Broadcast-only cost in 1996 dollars: $\$ 183334$, or $\$ 9.86$ per student.

Development and broadcast cost in 1996 dollars: $\$ 759436$, or $\$ 40.83$ per student

Life-years gained were discounted at $3 \%$.

$\mathrm{CI}=$ confidence interval
COST-EFFECTIVENESS RATIOS

Our estimates of the cost per life-year gained from the perspective of an organisation mounting a similar campaign in 1996 are shown in table 2 . These estimates show the cost per lifeyear gained without discounting the years, and with the years discounted at $3 \%$ and $5 \%$ over the life expectancy of young adult smokers. Based on the development and broadcast costs, the estimated cost per life-year gained, discounted at $3 \%$, would be $\$ 696$, and at $5 \%$, $\$ 1732$. For broadcast costs alone at these discount rates, the estimated cost per life-year gained would be $\$ 167$ and $\$ 417$, respectively. Table 2 also shows how the cost per life-year gained is influenced by the $95 \%$ confidence intervals of the observed-effect size of the mass media programme. The cost per life year gained ranges from about two-thirds to almost twice each estimate as the effect size varies from $7.8 \%$ to $3.2 \%$.

In addition to the development and broadcast costs, we examined the effect of higher costs. At $\$ 100$ per student, approximately 2.5 times the development and broadcast costs, the cost per life-year gained, discounted at $3 \%$, would be $\$ 1701$ (95\% CI = $\$ 1102$ to $\$ 2964)$.

We also examined the effect of reducing the difference in life expectancy between smokers and non-smokers. As would be expected, halving the difference to 2.53 years for young women and 3.16 years for young men, approximately doubles the cost per life-year gained. For development and broadcast costs, the estimated cost per life-year gained, discounted at $3 \%$, would be $\$ 1389$ (95\% CI = $\$ 900$ to $\$ 2420$ ), and for broadcast costs alone, $\$ 333(95 \% \mathrm{CI}=\$ 217$ to $\$ 598)$.

The prevalence of smoking increases into early adulthood, although less rapidly than during adolescence. ${ }^{32}$ Because our cohort of students became young adults in 1996, we examined the effect of a range of smoking prevalence on the cost-effectiveness ratios. We assumed that prevalence would change by the same proportion in both the mass media/ school programme communities and the school programme communities, and examined changes which ranged from 0.5 to 1.5 times the observed prevalence in the mass media/school programme and school programme communities. Table 3 shows how the cost-effectiveness ratio, discounted at $3 \%$, falls as the prevalence of smoking increases from $13 \%$ to $39 \%$, ranging from $\$ 1415$ to $\$ 464$ per life-year gained for developmental and broadcast costs, and from $\$ 335$ to $\$ 111$ per life-year gained for broadcast-only costs.

\section{COSTS FOR A NATIONAL PROGRAMME}

Table 4 shows the American student population aged 10-12 years in the areas of dominant influence; the costs of broadcasting the four-year media campaign in all large, medium, and small markets in the United States; and the costs per student potentially exposed to the campaign.

We estimate that a four-year national media campaign would cost about $\$ 83300000$ using 
Table 4 Population of students, air-time costs and costs per student for a national mass media smoking prevention campaign

\begin{tabular}{lrcrl}
\hline $\begin{array}{l}\text { Size of media } \\
\text { markets }\end{array}$ & $n^{*}$ & $\begin{array}{l}\text { Population of students } \\
\text { aged 10-12 years }\end{array}$ & $\begin{array}{l}\text { Four-year media campaign } \\
\text { air-time costs } \\
(\$)\end{array}$ & $\begin{array}{l}\text { Media costs per } \\
\text { student } \\
(\$)\end{array}$ \\
\hline Large & 40 & 6410600 & 55247000 & 8.64 \\
Medium & 80 & 2897600 & 19567000 & 6.76 \\
Small & 89 & 1094400 & 8477000 & 7.76 \\
Total & 209 & 10402600 & 83291000 & 8.01
\end{tabular}

$\star_{n}=$ Number of areas of dominant influence in each size market.

Table 5 Number of student smokers averted, and costs per student smoker averted, by a national mass media smoking prevention campaign

\begin{tabular}{lrrl}
$\begin{array}{l}\text { Size of media } \\
\text { markets }\end{array}$ & $n^{\star}$ & $\begin{array}{l}\text { Number of student } \\
\text { smokers averted }\end{array}$ & $\begin{array}{l}\text { Costs per student } \\
\text { smoker averted } \\
(\$)\end{array}$ \\
\hline Large & 40 & 320530 & 172 \\
Medium & 80 & 144880 & 135 \\
Small & 89 & 54720 & 155 \\
Total & 209 & 520130 & 160
\end{tabular}

$\star_{\mathrm{n}}=$ Number of areas of dominant influence in each size market.

${ }^{\star \star}$ An effect size of $5 \%$ was used to calculate the number of smokers averted.

1996 broadcast costs in all 209 American markets. Assuming the mass media campaign reduced the proportion of smokers by five percentage points, slightly less than we observed, table 5 shows the numbers of smokers averted in grades 10-12 and the costs per smoker averted.

The cost per student potentially exposed to this campaign would be about $\$ 8$. There would be about 520000 fewer smokers, at a cost of approximately $\$ 160$ per student smoker averted, and at a 3\% discount rate, $\$ 137$ (95\% $\mathrm{CI} \$ 89$ to $\$ 255)$ per life-year gained. To be culturally sensitive to students aged 10-15 years, throughout the United States, new television and radio spots would need to be produced, as the original spots were developed between 1985 and 1989 for a predominantly white student audience. If new television and radio spots were developed for audiences speaking English and Spanish, at an estimated cost of $\$ 1200000$, almost twice the cost of our 1985-89 campaign in 1996 dollars, this would increase the total cost by about $1.4 \%$, to approximately $\$ 84500000$ for a four-year campaign. The cost per student potentially exposed to this campaign would be $\$ 8.12$, the cost per student smoker averted, $\$ 162$, and the cost per life-year gained $\$ 138$ (95\% CI $=\$ 88$ to $\$ 252)$.

If such a campaign was broadcast nationally, and synchronised in all 209 markets, the efficacy of this campaign might approach that seen among the students who were fully exposed to the mass media campaign during the 1985-89 intervention trial. In that trial, in 1991 , we observed a reduction of 7.5 percentage points in the onset of smoking among those students who had been fully exposed to the media campaign and were still living in these communities two years after the campaign had ended. ${ }^{23}$ This would translate into about 780000 fewer smokers, and a corresponding reduction, to approximately $\$ 110$, in the cost per smoker averted in grades $10-12$, and $\$ 103$ (95\% CI $=\$ 67$ to $\$ 194)$ per life-year gained.
Discussion

This study shows that adding the mass media campaign to the school smoking prevention curriculum was economically attractive, that is, the incremental cost per life-year gained was relatively low. At a 3\% discount rate over the life expectancy of young adult smokers, a further 59 years for women and 50 years for men, the cost per life-year gained, \$696, compares favourably with the cost-effectiveness ratios of several smoking cessation interventions, and even more so when the broadcast costs alone are considered, $\$ 167$. For example, for brief smoking cessation advice from physicians provided during routine office visits, costeffectiveness ratios (in 1984 dollars) ranged for different age groups from $\$ 705$ to $\$ 988$ per year of life saved for men, and from $\$ 1204$ to $\$ 2058$ for women. ${ }^{4}$ For nicotine gum as an adjunct to physicians' advice, cost-effectiveness ratios (in 1984 dollars) ranged for different age groups from $\$ 4115$ to $\$ 6465$ per year of life saved for men, and from $\$ 6880$ to $\$ 9473$ for women. ${ }^{3}$ For the transdermal nicotine patch as an adjunct to physicians' advice, costeffectiveness ratios (in 1995 dollars) ranged for different age groups from $\$ 4390$ to $\$ 10943$ per quality-adjusted life years saved for men, and from $\$ 4955$ to $\$ 6983$ for women. ${ }^{5}$ Our estimates of the cost-effectiveness ratios of this mass media campaign are also less than those of several other preventive and therapeutic measures by up to three orders of magnitude. ${ }^{8918-20}$

The sensitivity analyses show that the incremental cost per life-year gained remains relatively low even with the low estimate for the effect size, $3.2 \%$, especially when considering the cost of broadcasting alone. Increasing the development and broadcasting costs to two and a half times the 1996 estimated cost increases the estimated cost per life-year gained to a similar extent. Halving the estimated difference in life expectancy of smokers compared with non-smokers almost doubles the cost-effectiveness ratio. With the same proportional increases in smoking prevalence into young adulthood in the mass media/ school programme and school programme communities, the cost-effectiveness ratios fall because the effect size increases. Most of these cost-effectiveness ratios, especially those related to the cost of broadcasting, are still lower than the cost-effectiveness ratios of the smoking cessation interventions noted above, ${ }^{3-5}$ even without adjusting those estimates to 1996 dollars.

Our estimate of the cost-effectiveness ratio of a four-year national campaign, $\$ 138$ per lifeyear gained, is based on our estimate of the number of 10-12-year-old students in all 209 media markets in the United States, and a reduction of five percentage points in the onset of smoking. We have not attempted to estimate the effect on either younger or older students who would also be potentially exposed to the mass media campaign, but it seems likely that our estimate of the cost effectiveness of such a campaign, by focusing on the cohort of 10-12year-old students, is conservative. 
For our estimates of cost-effectiveness ratios, we used the perspective of organisations interested in developing and implementing a similar mass media campaign. We have not included estimates of the future potential reduction in direct medical costs or indirect costs due to disability or premature death for the non-smokers resulting from our mass media campaign. Nor have we included estimates of the increased future costs of later medical care and for pensions and Social Security payments incurred as these non-smokers live longer. ${ }^{13}$ Our reasons for not including these costs spring partly from our perspective for estimating the cost effectiveness of the mass media campaign, but two other factors also played a part. First, there is considerable evidence from work comparing the lifetime costs of smokers and non-smokers that such future savings in direct and indirect costs are likely to be offset by the additional costs incurred as the non-smokers live longer, ${ }^{13}{ }^{34-36}$ although others have found that smokers incur higher costs. ${ }^{17}$ Second, when these costs have been included in a sensitivity analysis, as they were in Oster and colleagues' study of the cost-effectivness of nicotine gum as an adjunct to physicians' advice against cigarette smoking, they did not alter their results to any appreciable extent. ${ }^{3}$

Mass media have been used in four other campaigns aimed at preventing youth smoking. ${ }^{37-40}$ In one of these, the impact of mass media on adolescent smoking of a successful multi-component drug abuse prevention programme could not be assessed separately from the other components, precluding an estimation of cost effectiveness of the mass media. ${ }^{37}$ The other campaigns were unaccompanied by a reduction in youth smoking. ${ }^{38-40}$ The mass media campaign used as the model for the analyses reported here differed from these latter campaigns in several important respects: it used theoretically based educational objectives, targeted message development and delivery to specific audience segments, and resulted in intensive exposure to the messages throughout the period of greatest risk of smoking initiation. ${ }^{22}$

We conclude that estimates of the cost effectiveness of this four-year mass media campaign in preventing the onset of smoking show it to be economically attractive, with a relatively low incremental cost per life-year gained compared with other preventive and therapeutic strategies.

This project was supported by NIH grants CA38395 and CA22435.

1 Lynch BS, Bonnie RJ, eds. Growing up tobacco free: preventing nicotine addiction in children and youths. A report of the Committee on Preventing Nicotine Addiction in Children and Youths, Institute of Medicine. Washington, DC: National Academy Press, 1994:5.

2 Reid DJ, McNeill AD, Glynn TJ. Reducing the prevalence of smoking in youth in Western countries: an international review. Tobacco Control 1995;4:266-77.

3 Oster G, Huse DM, Delea TE, Colditz GA. Costeffectiveness of nicotine gum as an adjunct to physician's advice against cigarette smoking. $f A M A$ 1986;256:131518 .

4 Cummings SR, Rubin SM, Oster G. The cost-effectiveness of counseling smokers to quit. $¥ A M A 1989 ; 261: 75-9$.

5 Fiscella K, Franks P. Cost-effectiveness of the transdermal nicotine patch as an adjunct to physicians' smoking cessation counseling. $¥ A M A$ 1996;275:1247-51.
6 Kim S, Williams, Coleti SD, Hepler N, Crutchfield CC. Benefit-cost analysis of drug abuse prevention programs: macroscopic approach. $\mathcal{F}$ Drug Educ 1995;25:111-27.

7 Altman DG, Flora JA, Fortmann SP, Farquhar JW. The cost-effectiveness of three smoking cessation programs. Am f Public Health 1987;77:162-5.

8 Weinstein MC. Challenges for cost-effectiveness research. Med Decision Making 1986;6:194-8.

9 Oster G, Epstein AM. Cost-effectiveness of antihyperlipemic therapy in the prevention of coronary heart disease. pemic therapy in the prevent

10 Hammond EC. Life expectancy of American men in relation to their smoking habits. $\mathcal{F}$ Natl Cancer Inst 1969;43:951-62

11 Rogot E. Smoking and life expectancy among U.S. veterans. Am 7 Public Health 1978;68:1023-5.

12 Cohen BL, Lee I-S. A catalog of risks. Health Physics 1979, 36:707-22.

13 Warner KE. Health and economic implications of tobacco-free society. $7 A M A$ 1987;258:2080-6.

14 Taylor WC, Pass TM, Shepard DS, Komaroff AL. Cholesterol reduction and life expectancy: a model incorporating

15 Tsevat J, Weinstein MC, Williams LW, Toteson ANA, Goldman L. Expected gains in life expectancy from various coronary heart disease risk factor modifications. Circulation 1991;83:1194-201.

16 Rogers RG, Powell-Griner E. Life expectancies of cigarette smokers and nonsmokers in the United States. Soc Sci Med 1991;32:1151-9.

17 Hodgson TA. Cigarette smoking and lifetime medical expenditures. Milbank $Q$ 1992;70:81-125.

18 Stason WB, Weinstein MC. Allocation of resources to manage hypertension. New Engl f Med 1977;296:732-9.

19 Mushlin AI, Fintor L. Is screening for breast cancer cost-effective? Cancer 1992;69:1957-62.

20 Smith TJ, Hillner BE, Neighbors DM, McSorley PA, Le Chavalier T. Economic evaluation of a randomized clinical trial comparing vinorelbine, vinorelbine plus cisplatin, and vindesine plus cisplatin for non-small-cell lung cancer. $f$ Clin Oncol 1995;13:2166-73.

21 Flynn BS, Worden JK, Secker-Walker RH, et al. Prevention of cigarette smoking through mass media and school proof cigarette smoking through mass media a

22 Worden JK, Flynn BS, Geller BM, et al. Development of a smoking prevention mass media program using diagnostic and formative research. Prev Med 1988;17:531-58.

23 Flynn BS, Worden JK, Secker-Walker RH, et al. Cigarette smoking prevention effects on a cohort followed up two years after completion of mass media and school interventions. Am $\mathcal{F}$ Public Health 1994;84:1 148-51.

24 Worden JK, Flynn BS, Solomon LJ, et al. Preventing smoking among adolescent girls using mass media. Health Educ Q 1996;23:453-68.

25 Glynn TJ. School programs to prevent smoking: the National Cancer Institute guide to strategies that succeed. Bethesda, Cancer Institute guide to strategies that succeed. Bethesda,
Maryland: National Cancer Institute, Jan 1990. (NIH

26 Geller BM, Flynn BS. Smoking prevention curriculum. Burlington, Vermont: Office of Health Promotion Research, University of Vermont, 1992.

27 Economic report of the President. Transmitted to the Congress, February 1992. Washington, DC: US Government Printing Office, 1992.

28 Bowker RR. Broadcasting and cable yearbook 1994. New Providence, New Jersey: Reed Reference Publishing, 1994.

29 US census, 1990: census of population and housing (CD-ROM). Summary tape file $3 \mathrm{~A}$

30 Sonnenberg FA, Beck JR. Markov models in decision making a practical guide. Med Decision Making 1993;13:322-8.

31 Critchfield GC, Willard KE. Probabilistic analysis of decision trees using Monte Carlo simulations. Med Decision Making 1986;6:85-92

32 US Department of Health and Human Services. Preventing tobacco use among young people. A report of the Surgeon General. Atlanta, Georgia: Public Health Service, Centers for Disease Control and Prevention, Office on Smoking and Health, 1994 (US Government Printing Office No S/N Health, 1994 (US

33 Gilpin EA, Lee L, Evans N, Pierce JP. Smoking initiation rates in adults and minors: United States, 1944-1988. Am $\mathcal{F}$ Epidemiol 1994;140:535-43.

34 Leu RE, Schaub T. Does smoking increase medical care expenditure? Soc Sci Med 1983;17:1907-14

35 Leu RE, Schaub T. More on the impact of smoking on medical careexpenditures. Soc Sci Med 1985;21:825-7

36 Gori GB, Richter BJ, Yu WK. Economics and extended longevity: a case study. Prev Med 1984;13:396-410.

37 Pentz M, Dwyer J, MacKinnon, et al. A multicommunity trial for primary prevention of adolescent drug abuse effects on drug use prevalence. $\mathcal{F} A M A 1989 ; 261: 3259-66$.

38 Flay BR, Miller TQ, Hedeker D, et al. The television, school, and family smoking prevention and cessation project. Prev Med 1995;24:29-40.

39 Bauman KE, LaPrelle J, Brown JD, Koch CG, Padgett CA Influence of three mass media campaigns on variables related to adolescent smoking: results of a field experiment. Am f Public Health 1991;81:597-604.

40 Pierce JP, Evans N, Farkas AJ, et al. Tobacco use in California: an evaluation of the Tobacco Control Program, 1989-1993. Report to the California Department of Health Services. San Diego: University of California at San Diego, 1994:51-6. 